\title{
Philosophiques
}

\section{Réconciliation et éthique de la mémoire publique}

\section{Eamonn Callan}

Volume 29, numéro 2, automne 2002

La démocratie délibérative

URI : https://id.erudit.org/iderudit/006258ar

DOI : https://doi.org/10.7202/006258ar

Aller au sommaire du numéro

Éditeur(s)

Société de philosophie du Québec

ISSN

0316-2923 (imprimé)

1492-1391 (numérique)

Découvrir la revue

Citer cet article

Callan, E. (2002). Réconciliation et éthique de la mémoire publique.

Philosophiques, 29(2), 311-326. https://doi.org/10.7202/006258ar

\section{Résumé de l'article}

Cet article examine quelques idées concernant ce que j'appelle une éthique de la mémoire publique. Plus exactement, il considère comment les citoyens devraient apprendre à se souvenir du mal politique commis et subi dans des sociétés marquées par les circonstances de la réconciliation, dans lesquelles la stabilité politique et les pratiques démocratiques sont mises en péril par des souvenirs collectifs qui alimentent l'antipathie mutuelle et le ressentiment entre les citoyens d'un même État. Comment des communautés démocratiques fonctionnelles peuvent-elles être réparées ou créées à nouveau lorsqu'elles ont été fracturées par des hostilités historiques ? Cet article examine un certain nombre de réponses possibles à cette question en utilisant une conception délibérative de ce qu'une communauté démocratique fonctionnelle devrait être. Cette conception s'incarne en gros dans deux normes démocratiques : l'impartialité et l'inclusion. C'est en référence à ces deux normes que l'article discute différentes tentatives actuelles de remplacer les anciennes histoires nationales, qui s'appuyaient sur les mythes civiques, par des histoires multiculturelles qui les refusent.
Ce document est protégé par la loi sur le droit d'auteur. L'utilisation des services d'Érudit (y compris la reproduction) est assujettie à sa politique d'utilisation que vous pouvez consulter en ligne.

https://apropos.erudit.org/fr/usagers/politique-dutilisation/ 


\title{
Réconciliation et éthique de la mémoire publique
}

\author{
EAMONN CALLAN \\ Stanford University School of Education \\ ecallan@ stanford.edu
}

\begin{abstract}
RÉSUMÉ. - Cet article examine quelques idées concernant ce quej'appelle une éthique de la mémoire publique. Plus exactement, il considère comment les citoyens devraient apprendre à se souvenir du mal politique commis et subi dans des sociétés marquées par les circonstances de la réconciliation, dans lesquelles la stabilité politique et les pratiques démocratiques sont mises en péril par des souvenirs collectifs qui alimentent l'antipathie mutuelle et le ressentiment entre les citoyens d'un même État. Comment des communautés démocratiques fonctionnelles peuvent-elles être réparées ou créées à nouveau lorsqu'elles ont été fracturées par des hostilités historiques ? Cet article examine un certain nombre de réponses possibles à cette question en utilisant une conception délibérative de ce qu'une communauté démocratique fonctionnelle devrait être. Cette conception s'incarne en gros dans deux normes démocratiques : l'impartialité et l'inclusion. C'est en référence à ces deux normes que l'article discute différentes tentatives actuelles de remplacer les anciennes histoires nationales, qui s'appuyaient sur les mythes civiques, par des histoires multiculturelles qui les refusent.
\end{abstract}

\begin{abstract}
This article examines some ideas about what I call the ethics of public memory. M ore exactly, it considers how citizens should learn to remember political evil done and endured in societies marked by the circumstances of reconciliation in which political stability and democratic practices are thwarted by collective memories that fuel mutual antipathy and resentment among citizens of a common state. H ow democratically functional national communities can be repaired or created anew when they have been fractured by historical enmities? The article examines a number of possible answers using a deliberative conception of what a functional democratic community should be. This conception is roughly embodied in two democratic norms : impartiality and inclusion. It is in reference to those two norms that the article discusses current attempts at replacing the old national histories, which relied on civic myths, by multicultural histories, which eschew them.
\end{abstract}

1

Les frontières des États modernes ont été établies par des événements qui ont souvent transformé en concitoyens des gens déjà divisés par de profondes hostilités. Dans plusieurs cas, les hostilités ont été exacerbées par l'histoire subséquente. Ainsi, des mémoires collectives rivales sont transmises au fil des générations aux enfants qui, à la longue, transmettent à leurs propres enfants une hostilité qui participe à la constitution de leur identité. Dans ces conditions, la stabilité de tout État est vulnérable, et la coopération démocratique entre les différents groupes peut être presque impossible.

La puissance destructrice de l'hostilité ancestrale s'est avérée durable même lorsqu'il y a eu mélange des populations, avec pour conséquence que 


\section{2 · Philosophiques / Automne 2002}

la question de savoir à quel groupe tel ou tel doit être rattaché est de plus en plus arbitraire. Déjà la facilité avec laquelle la mémoire collective peut être utilisée comme élément déclencheur de la terreur est remarquable. Une faible dose de démagogie peut avoir des répercussions d'une ampleur étonnante en temps de crise politique ou économique ${ }^{1}$, et des récriminations historiques presqu'entièrement oubliées par plusieurs peuvent ressurgir avec violence et cruauté sur une échelle effroyable.

En fait, les récriminations historiques entre les groupes n'ont pas besoin d'être particulièrement anciennes pour dissoudre la coopération démocratique ou même les rudiments de l'ordre social. Une société antérieurement homogène sur le plan ethnique peut avoir été transformée en l'espace d'une génération par l'immigration. (L'immigration n'ayant pas besoin d'être une conséquence de la décolonisation, même si c'est fréquemment le cas.) Au sein de cette génération, les enfants d'immigrants grandissent avec les récits du mépris ou de la brutalité endurés par leurs parents en tant qu'étrangers indésirables dans cette société. Et avec le temps, ces enfants expérimenteront la même brutalité ou le même mépris. Ils n'ont jamais vécu ailleurs, mais ils en viennent à croire qu'ils demeureront toujours des étrangers indésirables. L'histoire de leur peuple au sein de l'État est brève mais douloureuse et leur rage envers ce qui semble être une exclusion permanente du plein statut de citoyen, souvent provoquée par leurs ennemis les plus xénophobes au sein de l'État, enflamme une violence épisodique et nourrit un désengagement politique désespérant.

II y a, enfin, des hostilités provoquées par les histoires récentes d'un gouvernement autocratique ou totalitaire. Les victimes de l'oppression vivent aux côtés de ceux qui ont perpétré ces abus ou en ont profité. Les complices des atrocités de l'ancien régime étaient trop nombreux pour être tous punis ou trop de torts ont été sanctionnés légalement au moment où ils étaient commis pour qu'une réparation soit possible devant les tribunaux. La justice rétributive peut être impossible à atteindre, et la responsabilité à l'égard des atrocités commises est souvent vigoureusement contestée. La dignité des victimes requiert pourtant une réparation sur le plan moral, et l'État semble irrévocablement entaché par le passé tant que rien n'est fait pour réparer les torts dont l'existence même avait été officiellement niée pendant si longtemps.

Les circonstances générales que je viens d'évoquer - appelons-les « les circonstances de la réconciliation » - sont des épreuves dans lesquelles la stabilité politique et les pratiques démocratiques sont perturbées par les mémoires collectives qui alimentent l'antipathie mutuelle et le ressentiment entre les citoyens d'un même État. Le conseil qui est habituellement donnéà

1. On a souvent fait la remarque ces dernières années à propos de la destruction causée par M ilosevic dans les Balkans : «Sans la propagande diffusée par Slobodan M ilosevic à propos de la façon dont les $M$ usulmans mangeaient des bébés serbes et prenaient des femmes serbes pour leurs harems, les Serbes ne se seraient pas retournés si brutalement contre leurs voisins». [Tina Rosenberg, "Afterword", dans M eredith, M artin, Coming to Terms : South Africa's Search for Truth, avec une préface et une postface par Tina Rosenberg, N ew York : Public Affairs, 1999.] 
une communauté aux prises avec ces circonstances est d'oublier le passé et de rétablir la vie politique sur de nouvelles bases. $M$ ais puisque la politique du présent ne peut être rien de plus que la politique que le passé rend possible, ce conseil est dénué de sens. Un nouveau départ en politique peut parfois nécessiter une rupture morale avec le passé, tout comme lorsque l'amnistie pour les meurtriers appartenant à des factions opposées s'impose comme le prix que chacun doit payer pour la paix. M ais si l'amnistie est un renoncement à la justice rétributive, elle n'autorise pas l'amnésie collective. Et se souvenir sincèrement du mal commis est l'une des tâches qu'il faut entreprendre si I'on veut s'assurer que le nouveau départ moral que l'on vise soit véritable.

Je veux esquisser quelques idées à propos de ce qui pourrait être appelé l'éthique de la mémoire publique. Plus précisément, je veux examiner comment les citoyens devraient apprendre à se souvenir du mal politique commis et subi dans les sociétés marquées par les circonstances de la réconciliation. Je suppose que ces circonstances doivent être comprises dans le contexte d'un État moderne ou d'États multinationaux dans lesquels la sécession ou la redéfinition des frontières politiques est rarement réalisable. Je suppose également que les institutions politiques internationales qui pourraient dépouiller les États d'une bonne partie de leur autorité actuelle et les nations d'une bonne partie de leur emprise sur l'imaginaire populaire restent toujours pour la plupart une lointaine perspective. Ces données de départ requièrent que nous réféchissions sur la façon dont des communautés nationales fonctionnelles démocratiquement peuvent être réparées ou créées à nouveau lorsqu'elles ont été fracturées par des hostilités historiques. La réconciliation, en d'autres mots, est un type de construction de la nation même si certains des torts qu'elle s'efforce de rétablir sont souvent le legs de projets antérieurs de construction de la nation.

\section{2}

La réconciliation politique requiert la résolution du conflit politique. $M$ ais toute résolution n'est pas réconciliation. Ceux qui éprouvent un ressentiment amer devant la défaite de leur cause ne peuvent se réconcilier avec ceux qui I'ont assurée, même lorsque le conflit s'est terminé depuis longtemps. La réconciliation personnelle consiste dans le rétablissement d'une amitié perdue, et son analogue politique doit consister en des liens d'amitié civique ou de solidarité à recouvrer ou à recréer à nouveau à la suite du conflit. La guerre civile américaine a pris rétrospectivement la forme d'un conflit fratricide, même s'il n'y avait rien de particulièrement fraternel dans les relations entre combattants. La fiction d'une fraternité rompue était un moyen avantageux de réinterpréter les antipathies violentes de la guerre d'une façon qui résonne d'une nouvelle aspiration partagée à rétablir la coopération sociale entre les populations blanches du N ord et du Sud².

2. Blight, David W., Race and Reunion : the Civil War in A merican M emory, Cambridge, M ass. : H arvard University Press, 2001. 


\section{4 - Philosophiques / Automne 2002}

Cette réconciliation en particulier est révélatrice pour une autre raison. La subordination systématique des $\mathrm{N}$ oirs théoriquement affranchis sous les lois et coutumes de «Jim C row » était solidement établie à travers les États du Sud dans la dernière décennie du XIX ${ }^{e}$ siècle. Ce dispositif a joué un rôle crucial dans la consolidation de l'unité de l'État-nation américain moderne, en dépit de son caractère terriblement oppresseur. La réconciliation peut donc être réalisée à des coûts qui sont moralement répugnants, même si refuser d'assumer ces coûts perpétue le conflit et la coercition. Cela étant, les appels aux valeurs de la réconciliation sont vides en l'absence de clarté quant au caractère substantiel de la communauté politique dans laquelle ceux qui sont actuellement divisés sont supposés atteindre la solidarité.

Cela m'oblige à dire quelques mots à propos de la façon dont je comprends l'idée d'une communauté démocratique fonctionnelle. Je suppose que la politique, dans le meilleur des cas possibles, doit réaliser deux normes démocratiques qui nous sont familières, et que celles-ci auront prise sur la culture civique de toute communauté de ce genre. Premièrement, je suppose qu'un débat politique est un débat moral entre égaux à propos de l'exercice du pouvoir public. II est délibératif plutôt que stratégique. Cela présuppose que nous soyons capables de faire la différence de façon plus ou moins fiable entre des intérêts particuliers ou privés, d'une part, et le bien commun et les droits des individus ou des groupes, d'autre part. Le débat politique comporte ainsi une norme d'impartialité: les intérêts de tous ceux qui font partie de la communauté des citoyens doivent être considérés sans préjugé ou parti-pris dans la prise de décisions politiques collectives. Deuxièmement, la délibération implique une norme d'inclusion : les arguments moraux opposés que les citoyens formulent en toute bonne foi dans la délibération doivent être scrupuleusement examinés et le compromis mutuel doit être recherché. Les valeurs publiques seront interprétées de plusieurs façons différentes bien que raisonnables à l'intérieur du donnant-donnant de la vie politique démocratique, et ce même si le consensus demeure le but recherché. Idéalement, les conceptions de chacun doivent être respectées et considérées sérieusement, et même de vagues approximations de cet idéal généreront inévitablement conflits et désaccords que le respect mutuel exige que nous concilions.

II pourrait sembler au mieux arbitraire de lier le problème de la réconciliation avec l'idéal d'une communauté qui soit démocratique et délibérative. A près tout, la critique peut-être la plus commune formulée contre cet idéal est qu'il est utopique dans le mauvais sens du terme. La politique démocratique ordinaire est trop profondément pénétrée par des conflits d'intérêts individuels et trop peu influencée par les valeurs morales pour que la démocratie délibérative soit plus qu'un rêve chevaleresque. Et si cela est vrai de la politique lorsque des conflits d'intérêts relativement modestes sont en jeu, l'exigence morale de la délibération pourrait sembler particulièrement absurde dans les circonstances de la réconciliation, où le ressentiment et la haine intensifient fortement les frictions habituelles de la vie publique. 
Pourtant, en y regardant de plus près, toute interprétation de la démocratie qui abandonne les revendications morales d'inclusion et d'impartialité est précisément ce dont les circonstances de la réconciliation montrent l'absurdité. Le langage que nous employons pour affronter ces circonstances est irréductiblement moral - on parle de la victoire sur l'oppression, de restitution, de rétribution, ainsi de suite, et l'ampleur de ce qui est en jeu teinte la façon dont les groupes de citoyens se perçoivent les uns les autres ainsi que la manière dont ils évaluent les risques de la coopération. Une vision réductrice du jugement moral en politique semble plausible lorsque nous nous attardons aux querelles triviales des groupes d'intérêts, lorsque le conflit survient dans un contexte d'institutions démocratiques stables et relativement justes que l'on tient pour acquis. A près tout, seule une incroyable naïveté porterait quelqu'un à supposer que le débat «moral » en politique n'est pas le plus souvent un genre de sophistique dans lequel l'intérêt particulier des agents publics, suivant astucieusement le contour des intérêts de ceux qu'ils représentent, est avancé sous l'apparence d'un principe moral et noble. Ainsi, tant qu'aucun droit fondamental n'est violé, accepter cette sophistique comme une donnée incontournable de la vie politique pourrait sembler n'être que le simple bon sens. $M$ ais la gravité de ce que nous considérons comme étant en jeu dans les circonstances de la réconciliation, plus particulièrement lorsqu'on se trouve parmi les victimes de l'oppression ou que l'on adhère à leur cause, met en évidence une vision de la politique démocratique à laquelle sont inextricablement attachées des attentes d'un ordre fondamentalement moral. Je pense ici aux attentes concernant l'exercice d'un pouvoir qui soit limité par des normes morales assurant l'égalité des citoyens et protégeant les minorités de tout abus. Et parce qu'une politique de l'intérêt particulier ne peut rencontrer ces attentes de façon fiable, nous sommes conduits vers une conception de la communauté démocratique dans laquelle la délibération commune l'emporte sur toute stratégie de faction.

Une communauté démocratique réalisable dans laquelle les normes d'inclusion et d'impartialité sont généralement satisfaites contiendra aussi de fortes tendances qui la poussent vers d'autres directions, et il serait utopique dans la pire acception du terme de supposer qu'il puisse en être autrement. $\mathrm{Ce}$ qui rend ces tendances anti-délibératives particulièrement redoutables dans les circonstances de la réconciliation c'est qu'elles sont typiquement liées avec les forces mythiques et mobilisatrices de la nation elle-même.

\section{3}

Les nations sont des communautés politiques intergénérationnelles. Elles sont liées à travers le temps par une identification commune à la communauté, par la conviction partagée que les membres entretiennent des obligations particulières les uns envers les autres en vertu de leur appartenance et par une prétention plus ou moins consensuelleà l'autodétermination politique. Les arguments que les citoyens présentent les uns aux autres en tant que membres d'une même 


\section{6 - Philosophiques / Automne 2002}

nation sont des arguments portant sur l'usage du pouvoir partagé qui appartient légitimement à leur communauté politique. La communauté est donc ce qui est dénoté par le préfixe « auto » dans l'idée d'autodétermination nationale. Rien de métaphysiquement substantiel n'est impliqué par cette phrase : tout ce qui est connoté est l'identification aux autres dans le projet collectif de la nation. $M$ ais précisément parce que les arguments qu'échangent les citoyens appartiennent à la vie commune d'un projet collectif intergénérationnel, ils doivent faire appel à une orientation partagée au passé, d'où est né ce projet et qui lui donne son élan. C'est une telle mémoire collective qui rend, pour ainsi dire, le débat public mutuellement compréhensible et, dans la plupart des cas, réciproquement acceptable.

L'idée d'une mémoire civique implique une identité collective de la même manière que l'idée d'auto-gouvernement démocratique. $N$ os souvenirs individuels - incluant ceux qui influent sur les rôles que nous jouons en tant que citoyens particuliers - ne sont pas les mêmes que ces souvenirs qui resaisissent le sens public du passé que nous possédons en commun en tant que membres d'une communauté nationale. Le nom d'un ami ou d'un membre de la famille sur le Vietnam M emorial à Washington suscite un souvenir personnel et le chagrin privé qui lui est associé. Pourtant, le mur en lui-même, avec son énumération sobre et implacable de tous les Américains morts au cours de cette guerre, évoque la mémoire publique et la tristesse d'une nation envers les soldats qu'elle a perdus dans une guerre qui n'a toujours pas de signification publique partagée. La mémoire nationale est créée et soutenue par des choses comme celles-ci : les monuments publics, les noms de rues, I'histoire politique enseignée dans les écoles, les traditions apprises en famille, les rituels civiques, les musées, les sites historiques, et, bien sûr, ces moments où les politiciens démocratiques peuvent assumer effectivement une posture singulièrement solennelle et exaltée et insérer leurs ambitions dans le grand récit de la nation elle-même.

Benedict Anderson a fait l'affirmation devenue fameuse que toutes les nations sont des «communautés imaginées». Cela veut dire que les nations sont rendues possibles à travers le pouvoir constructif de l'imagination. Les êtres humains ne rencontreront directement qu'une infime partie seulement de ceux qui partagent avec eux ces représentations imaginées de la nation, et pourtant ce sont ces mêmes représentations qui constituent la nation. «Un A méricain ne rencontrera ni ne connaîtra jamais plus qu'une poignée de ses quelques 240000 concitoyens A méricains. II n'a aucune idée de ce qu'ils peuvent projeter à un moment quelconque. $M$ ais il a une entière confiance dans leur activité assidue, anonyme et simultanée» ${ }^{3}$. La nation ainsi «imaginée» n'est pas simplement imaginaire, pas plus que ne le sont d'autres collectivités à grande échelle dans lesquelles les gens s'identifient de façon

3. Anderson, Benedict, Imagined Communities : R eflections on the 0 rigins and Spread of $\mathrm{N}$ ationalism, nouvelle éd., London : Verso, 1991, p. 26. 
consciente. Cependant Anderson veut également suggérer que l'imaginaire est profondément impliqué dans l'être même des nations. II souhaite, en effet, nous convaincre non seulement du fait qu'une mémoire publique commune est nécessaire à toute citoyenneté nationale, mais que le contenu de cette mémoire doit remplir les fonctions influentes, porteuses de sens et mobilisatrices du mythe social.

L'argument d'A nderson sur ce point est basé en partie sur l'idée qu'une histoire nationale mythique remplit le vide psychologique créé par la sécularisation rationaliste propre à la modernité : «La désintégration du paradis : rien ne rend la fatalité plus arbitraire. L'absurdité du salut : rien ne rend un autre type de continuité plus nécessaire. Ce dont on avait besoin alors était une transformation laïque de la fatalité en continuité, de la contingence en sens ${ }^{4}$. La grande nation a rempli cette fonction. $M$ ais ceci n'est qu'une demivérité révélatrice. A près tout, la religion a servi aussi souvent de repère local déterminant de la nationalité qu'elle n'a été cette source vivante de mythe que le nationalisme al lait remplacer. Q uoi qu'il en soit, le fondement mythique commun de la nation et de la religion est évident dans l'intensité et le caractère récalcitrant que la mémoire nationale collective peut manifester, dans la puissance émotive que ses récits de gloire patriotique et de martyre peut exercer, ainsi que dans la force avec laquelle elle relie les grandeurs et tragédies anciennes à des avenirs utopiques. Le problème est alors que les enjeux du dialogue entre groupes à l'intérieur des États-nations qui font face à de sérieux problèmes de réconciliation deviennent dangereusement importants. Ce n'est pas simplement que des torts anciens doivent être réparés; c'est que des identités anciennes doivent être reconstruites lorsque leurs sources mythiques traditionnelles sont mises en cause.

\section{4}

Je soutiens que l'éthique de la mémoire publique qui sied aux circonstances de la réconciliation requiert l'adoption d'une attitude sceptique quant aux structures caractéristiques du mythe national. En même temps, je soutiens que la démolition du mythe national peut elle-même prêter le flanc à certains maux civiques auxquels une mémoire publique civique résistera.

Pour qu'une nation survive et prospère, les gens ont besoin de raisons à la fois pour soutenir la communauté imaginée qui en fait des co-nationaux et pour faire des sacrifices lorsque cela est nécessaire pour le bien de la nation. La source de telles raisons a été trouvée traditionnellement dans un passé mythique - ce qu'A nthony Smith nomme l'«âge d'or » - lorsque le caractère distinct de l'identité nationale a été déterminé à travers des événements et des réalisations d'une signification importante et durable ${ }^{5}$. En plus des

4. Ibid., p. 11.

5. Smith, Anthony, $\mathrm{M}$ yths and $\mathrm{M}$ emories of the $\mathrm{N}$ ation, $\mathrm{O}$ ford : Oxford University Press, 1999, pp. 65-8, 261-5. 


\section{8 · Philosophiques / Automne 2002}

sacrifices et batailles épiques, de tels souvenirs incluent « les mouvements religieux et leurs dirigeants, les migrations, les découvertes et colonisations, la fondation de villes et d'États, les dynasties et leurs rois, les codes de lois et leurs législateurs, les grandes constructions et leurs architectes, les peintres, les sculpteurs, les poètes, les musiciens et leurs oeuvres immortelles». Sel on Smith, le passé national mythique "en vient à définir le caractère normatif d'une communauté en évolution. » Les acteurs historiques deviennent des héros dont l'exemple incite à l'émulation, et les citoyens sont honorés et inspirés par la perspective d'un « potentiel par la filiation » parce que les liens de sang qui les rattachent aux générations antérieures deviennent la promesse d'une gloire et d'un accomplissement futurs ${ }^{6}$. M ême aux États-Unis, société d'immigration par excellence, les attraits du «potentiel par la filiation» ont souvent été irrésistibles. Tel que l'a montré J onathan Zimmerman, lorsque des immigrants d'Irlande, de même que ceux d'Europe du Sud et de l'Est, ont réclamé la révision de l'histoire dans les écoles américaines au début du vingtième siècle, c'était pour faire une place aux héros irlandais, italiens ou polonais au sein du Panthéon de la Révolution américaine; ce n'était pas pour remettre en question le mythe reçu de la Révolution lui-même?.

Les théoriciens politiques perçoivent souvent le contenu mythique de la mémoire collective de façon quel que peu indulgente. Par exemple, dans l'étude magistrale de Rogers Smith sur le droit américain de la citoyenneté, Civic Ideals, un rôle nécessaire est donné au mythe dans les politiques des Étatsnations. D'une part, les exigences de l'exercice du pouvoir politique, même sous des conditions démocratiques favorables, rendent nécessaire l'éveil et le soutien du sentiment que l'ensemble des citoyens constitue un peuple spécial et distinct. Sans une identité valorisée et partagée, I'appui des citoyens ne peut être obtenu, et cette identité commune ne peut être réalisée sans la construction du mythe civique. À l'instar de James M adison, Smith remarque qu'aucune nation n'a établi son identité à partir d'idéaux purement philosophiques et que les mythes qui disent aux citoyens qu'ils sont « choisis et bénis» sont de puissants moyens pour inculquer la loyauté et le respect des lois. Le mythe civique conforte les citoyens dans l'idée que leur appartenance politique est « intrinsèquement juste et bonne» et permet à plusieurs de ceux qui sont en position d'infériorité de se sentir comme faisant partie d'une «plus vaste, plus forte unité ayant une valeur intrinsèque qui continuera de s'épanouir après qu'ils aient péri, de sorte qu'ils n'auront pas vécu en vain ${ }^{8}$.

Le mythe civique a donc, sel on Smith, des fonctions cruciales : mobilisatrice et herméneutique. II fournit à la fois le cadre interprétatif à l'intérieur duquel les responsabilités et vertus de la citoyenneté doivent être comprises

6. Ibid., p. 262-263.

7. Zimmerman, Jonathan. "Each "Race" Could $\mathrm{H}$ ave Its H eroes Sung: Ethnicity and the $\mathrm{H}$ istory Wars in the 1920s», The J ournal of A merican H istory, 87, 2000.

8. Smith, Rogers, Civic I deals : Conflicting Visions of Citizenship in US H istory, $\mathrm{N}$ ew H aven : Yale University Press, 1997, p. 35-9. 
ainsi que les raisons motivant l'acquittement de ces responsabilités et la culture de ces vertus. Smith est très sensible au potentiel oppressif du mythe civique, alors même qu'il insiste sur sa nécessité politique. Toutefois, il ne tient pas compte de certaines pathologies qui lui sont endémiques. Plus particulièrement, il ferme les yeux sur les polarisations morales corruptrices que crée le mythe.

Le mythe civique forme - et est formé par - les idéaux politiques et les partis-pris idéologiques, mais la distinction entre le mythe et l'idéal est de première importance. La doctrine des droits de l'homme est un idéal qui permet un ordonnancement de la vie qui peut guider la critique sociale et morale sans masquer à quiconque la dense, ambiguë et immanquablement surprenante particularité du monde humain réel. $M$ ais dans le mythe civique, les idéaux deviennent incarnés lorsque les fondateurs ancestraux sont transformés en personnifications du bien et du juste, et que les ironies embrouillées du changement et du conflit historique sont effacées dans les grandes lignes du récit épique. Les polarités créées par le récit mythique impliquent une perte de finesse du jugement politique et rendent impossible toute critique humaine et libre de préjugés dont dépend une compréhension suffisamment fine de la justice et de l'oppression dans I'histoire. Si la vertu héroïque doit briller d'un éclat mythique, alors le vice - ou plus précisément quiconque est décrit comme s'opposant à ceux qui personnifient la vertu réalisée ou représentée - doit être démonisé. Si la nation doit être sacralisée par la pureté morale de ses héros et les souffrances de tous ceux qui ont souffert en son nom, alors ses adversaires doivent subir une transformation symétrique pour incarner le mal.

Voilà qui me conduit à mon propos principal. Les conflits qui marquent les circonstances de la réconciliation indiquent la présence de crises dans les mythes nationaux dominants. Ils exposent, en effet, les ambiguïtés morales (ou pire) des réalisations de la nation ou mettent en cause des pratiques vénérées que le mythe prétend légitimer. Les groupes marginalisés ou mal représentés par le mythe dominant revendiquent ce qui leur revient, et précisément parce que le mythe dominant est déterminant pour l'identité de tant d'individus, les enjeux du conflit sont extrêmement élevés. $M$ ais la dissolution du mythe national est critique parce qu'aussi longtemps que son pouvoir est maintenu nous ne pouvons attendre du discours politique qu'il honore les prétentions à l'impartialité et à l'inclusion. Le mythe glorifie les intérêts de ses héritiers aux dépens de ceux qui ne peuvent prétendre au « potentiel par la filiation » conféré par le mythe. Plus encore, le besoin d'établir un récit mythique unique faisant autorité restreint inévitablement la pluralité des perspectives et interprétations raisonnables que toute histoire commune riche et complexe évoquera.

Supposons que nous acceptions que le travail de réconciliation requière une mémoire publique démythifiée. La question se pose alors de savoir quel genre de mémoire publique devrait la remplacer. R appelons-nous que le mythe national remplit une fonction mobilisatrice et interprétative dans les démo- 


\section{$320 \cdot$ Philosophiques / Automne 2002}

craties de masse. II permet ce que A nderson appelle « la camaraderie profonde et horizontale» de l'appartenance commune à la nation. Les circonstances de la réconciliation indiquent des ruptures profondes dans ce lien, mais si la réconciliation doit avoir lieu, ce lien doit être rétabli. Est-ce qu'une mémoire publique démythifiée peut produire ce même résultat? Un cas intéressant qui permet de répondre oui - ou du moins de répondre oui dans le contexte américain contemporain - se trouve dans la défense vigoureuse que fait $\mathrm{G}$ ary $\mathrm{N}$ ash d'un patriotisme démocratique post-mythique dans son ouvrage fort intéressant : History on Trial ${ }^{9}$.

\section{5}

$\mathrm{N}$ ash est parmi les historiens américains les plus réputés, et son argumentation est présentée comme une défense des tentatives récentes pour ajuster l'enseignement de l'histoire dans les écoles élémentaires et secondaires aux connaissances contemporaines et multiculturelles de l'histoire de la nation. J'insisterai particulièrement sur cet exemple parce qu'il illustre plusieurs des problèmes qui minent les efforts pour s'approprier l'histoire dans un esprit de réconciliation.

Pour la grande part de leur histoire, les écoles américaines ont pu, avec une relative facilité, jeter un voile sur les éléments de racisme et sur le caractère oligarchique du mythe dans le récit patriotique de la nation. L'historiographie universitaire américaine n'a pas brusquement et systématiquement divergé de l'histoire patriotique racontée dans les écoles, et ainsi le mythe enseigné aux enfants pouvait revendiquer la légitimité des connaissances de l'époque. Plus important encore, les voix politiques de ceux que ce récit excluait d'une égale citoyenneté demeuraient généralement muettes ou inaudibles. Tout cela a changé. L'historiographie universitaire américaine s'est développée dans les dernières décennies dans des directions qui discréditent plusieurs éléments du récit patriotique traditionnel ${ }^{10}$. L'esclavage n'était pas l'institution bénigne et paternaliste telle qu'on l'avait généralement présentée pour la plus grande partie du vingtième siècle; elle était l'oppression incarnée dans ce qu'elle a de plus terrible. Et l'Émancipation n'a pas sonné la fin de tous les torts résultant de l'esclavage; ce n'était qu'un épisode au sein de la lutte intermittente et toujours incomplète contre le racisme américain. Ces tournants dans I'historiographie universitaire reflètent des changements plus importants dans le corps politique qui semblent maintenant irréversibles. D es minorités autrefois muettes revendiquent l'égal ité et la reconnaissance. Et le lent mais inexorable déclin démographique des Blancs américains vers un statut de minorité parmi d'autres commence à s'inscrire dans la conscience populaire, rendant plus difficile pour quiconque de supposer que l'identité

9. N ash, Gary B., Crabtree, Charlotte and Dunn, Ross E., History on Trial : Culture Wars and the Teaching of the Past, 2e éd., N ew York : Vintage, 2000.

10. Ibid., p. 76-82. 
américaine puisse être simplement l'identité blanche. La question est alors de savoir comment la nouvelle histoire multiculturelle pourrait nourrir la mémoire publique nécessaire à la réconciliation raciale aux États-U nis.

Selon $\mathrm{N}$ ash, l'enseignement de la nouvelle histoire ne remplace pas le patriotisme par autre chose; il signale plutôt la transition à partir d'un ancien patriotisme, biaisé par le mythe intéressé de l'élite anglo-saxonne, vers une nouvelle version plus égalitaire qui rend les honneurs dus à tous ceux qui ont fait de l'A mérique ce qu'elle est aujourd'hui ${ }^{11}$. L'histoire multiculturelle explore le passé à partir des nombreuses perspectives différentes de ceux dont les vies étaient invisibles dans l'ancienne histoire patriotique des grands hommes, des grandes guerres et du progrès moral continu. $N$ ous avons maintenant une histoire qui procède autant du bas vers le haut que du haut vers le bas - I'histoire des pauvres comme celle des riches, des femmes comme celles des hommes. $M$ ais même ces distinctions sont beaucoup trop simples: l'A mérique vécue par les femmes immigrantes n'était pas la même, quelle que soit leur provenance ou leur destination, et être une femme noire n'a jamais été l'équivalent d'être une femme blanche, et ainsi de suite. Et ces catégories perdront également leur apparente cohérence sous un examen minutieux alors que les différences à l'intérieur de chacune d'elles se préciseront. Les perspectives prolifèrent donc dans I'environnement universitaire là où la dissolution des catégories unitaires est la stratégie intellectuelle favorisée, et alors qu'elles se dissolvent, la possibilité de toute histoire nationale englobante pourrait sembler de plus en plus hors d'atteinte.

S'il y a là un triomphe de l'inclusion, cela est moins clair en revanche pour l'impartialité. La multiplication des perspectives historiques ne signifie pas nécessairement, en effet, qu'une mémoire publique plus impartiale, que les citoyens pourraient adopter ensemble, soit rendue possible par cette nouvelle historiographie. Cette prolifération pourrait simplement signifier que plusieurs points de vue partiaux et incommensurables ont été construits, et que l'addition de ces partialités multiples n'a pas nécessairement pour résultat l'impartialité. Le fait que certaines d'entre elles expriment les préoccupations de groupes antérieurement ou actuellement opprimés n'est pas une preuve de leur supériorité au plan épistémique. Supposer que l'oppression ou l'exclusion confère à certains une clairvoyance interdite aux autres n'est que du sentimentalisme. On pourrait donc s'inquiéter qu'aucun cadre herméneutique commun émerge de ce mélange de voix et de perspectives rivales. Si aucun cadre de ce type n'émerge, alors l'espoir qu'une histoire démythifiée puisse apporter quoi que ce soit au progrès civique serait simplement anachronique.

$N$ ash est conscient de cette difficulté, et dans une ébauche interprétative de la Révolution américaine, il tente de montrer comment les nouvelles connaissances historiques offrent une lecture des débuts de la R épublique de loin plus compliquée, et plus conflictuelle, mais néanmoins patriotique. Selon

11. Ibid., p. 15. 


\section{2 - Philosophiques / Automne 2002}

$N$ ash, la R évolution a été le lieu de multiples batailles pour la liberté dont certaines furent des échecs tragiques. «Les N oirs américains se sont joints à des alliés Blancs (i.e. britanniques) pour montrer la contradiction entre la résistance à l'oppression britannique et la réalité de l'esclavage». Les A mérindiens qui n'ont rien gagné à se battre ont manifesté « leur désir constant de proté ger la vie, la liberté et la quête du bonheur proclamés par la nouvelle république». Et dans la dispute entre A bigail Adams et son mari John Adams quant aux maux supposés de l'autorité patriarcale, $\mathrm{N}$ ash détecte les premiers remous d'un processus qui devait mener éventuellement au « suffrage et [au] traitement légal équitable des femmes ${ }^{12}$. En un mot, la Révolution a été une lutte pour la liberté dans laquelle tous les Américains ont participé d'une façon ou d'une autre, même si la liberté de certains est advenue au prix de l'assujettissement de certains autres.

La rapide esquisse de la Révolution que nous donne $N$ ash inclut beaucoup trop de groupes dont la liberté a été brimée pour qu'on puisse inférer qu'une antipathie de principe à l'égard de l'oppression en a été la force motrice. $N$ ash serait presque assurément d'accord avec nous sur ce point. M ais pourquoi alors le cadre interprétatif de la liberté pour tous, qu'il recommande pour l'écriture d'une histoire multiculturelle de l'A mérique, devrait-il conduire à un nouveau patriotisme américain? La réponse de $\mathrm{N}$ ash est esquissée dans ce qu'il dit de la «contradiction» entre le noble fondement moral de la citoyenneté libre et égale à partir duquel les colons ont établi leurs revendications et l'oppression dans laquelle ces mêmes colons étaient profondément impliqués ${ }^{13}$. Le leitmotiv de la nouvelle histoire patriotique devient alors les idéaux élevés de la citoyenneté libre et égale au cœur de l'accord constitutionnel américain, ainsi que les trahisons que ces idéaux ont subies, et les nombreuses luttes toujours en cours pour les rendre réels dans la vie de tous les citoyens.

Cela est fort éclairant, mais laisse quelques questions importantes en suspens. Pris en lui-même, le cadre interprétatif que $\mathrm{N}$ ash voudrait faire adopter à nos enfants peut mener autant à l'apathie civique ou au désespoir qu'à une citoyenneté engagée et démocratique. Pour que l'histoire nourrisse l'engagement patriotique, il doit y avoir une identification antérieure aux idéaux politico-moraux qui guident le cadre interprétatif de $\mathrm{N}$ ash, de même que de fortes raisons pour que les étudiants croient que la «contradiction » entre l'idéal et la réalité n'est pas importante et inébranlable au point de retirer à leur citoyenneté toute signification réelle. Faire face au contexte historique dans lequel la réconciliation doit être recherchée implique communément une confrontation avec le caractère apparemment irrémédiable d'un très grand mal, et si cela ranime de puissantes émotions publiques, celles-ci peuvent prendre autant la forme d'une horreur paralysante ou d'un dégoût, que d'un enthousiasme pour rebâtir la nation de nouveau.

12. Ibid., p. 84-6.

13. Ibid., p. 85. 
La question est de savoir si une mémoire publique démythifiée peut soutenir de solides espoirs civiques. Sur cette question, pourtant, I'historiographie universitaire américaine dans son état actuel ne parle pas d'une voix uniformément encourageante, et dire que la nouvelle histoire multiculturelle enseignera un patriotisme aussi inspirant que l'ancien est peut-être un peu facile. Considérons une polémique récente dans les pages du Journal of American $\mathrm{H}$ istory. Gary Gerstle, un historien respecté de I'Université du $M$ aryland, répond à un article de $D$ avid $H$ ollinger, auteur d'un livre acclamé à juste titre et qui porte un regard avisé et optimiste sur l'avenir du sentiment national A méricain (I'article de H ollinger était lui-même une réplique à une critique antérieure faite par G erstle) :

$\mathrm{H}$ ollinger me critique pour avoir négligé la distinction entre sa description du passé, plein de coercition et de limites, et son esquisse «normative» du futur, dans laquelle il imagine une Amérique libre de telles contraintes. Toutefois la confusion ne vient pas de moi, mais de lui ; si le passé a été si coercitif, pourquoi le futur devrait-il être si différent? (...) N otre histoire suggère que bâtir une communauté nationale repose sur la répression et l'exclusion. (mes italiques) ${ }^{14}$

Gerstle avait exprimé ailleurs ses appréhensions quant au nationalisme américain avec une plus grande circonspection ${ }^{15}$. M ais ce passage est particulièrement révélateur. Pour tous ceux qui ont portéla moindre attention aux sciences sociales et aux humanités sur les campus américains ces dernières années, il a un caractère familier. II est familier non pas parce qu'il est typique du discours propre aux humanités et aux sciences sociales, mais plutôt parce qu'il est une variété présentement en vogue à l'intérieur du genre universitaire de la vanité et de l'arrogance. Le spécialiste emprunte la pause du moraliste vertueux et entièrement sûr de lui-même, juge du passé, du présent et du futur national. (J e soupçonne que cette attitude est l'une des plus étranges mutations de ce vénérable archétype américain - le prédicateur puritain et moralisateur.) Et ce moraliste a de mauvaises nouvelles à nous apprendre : le passé a été terrible, le présent est tout aussi mauvais et le futur le sera également. Les autres (i.e. les spécialistes qui s'opposent à cette vision) sont simplement trop peu vertueux ou perspicaces pour le voir.

Cela constitue le summum de l'histoire antipatriotique. Accepter ce noble désespoir revient à abandonner l'idée même de bâtir une communauté nationale. $M$ ais se résoudre à cela c'est aussi abandonner nécessairement la construction d'une communauté nationale démocratique. Bien sûr, Gerstle considère la démocratie comme une très bonne chose. Pourtant, la cohérence requerrait qu'il nous dise que la seule démocratie authentique pour les A méricains ne pourrait être pratiquée que dans une A mérique «post-nationale», dans laquelle

14. Gerstle, Gary, «The Power of N ation », The J ournal of A merican H istory, 85, 1997, p. 576.

15. Gerstle, Gary, American Crucible : Race and N ation in the Twentieth Century, Princeton, N J : Princeton University Press, 2001, p. 373-4. 


\section{4 - Philosophiques / Automne 2002}

la réconciliation a été abandonnée. C'est dire que les groupes qui ont été les victimes de l'oppression ne devraient plus chercher à atteindre aucune « communauté imaginée» commune d'auto-gouvernement avec ceux qui ont été responsables de leur oppression. L'État américain pourrait continuer à exister. $M$ ais pour tous ceux qui ont abjuré la nation, il ne pourrait pas être l'instrument d'une communauté démocratique partagée; il ne serait probablement que le lieu d'une lutte pour gagner un pouvoir devant être utilisé contre ceux qui voudraient exploiter ces groupes pour leurs propres fins. Cela serait assurément un type d'A mérique multiculturelle : la « différence» serait victorieuse contre l'«uniformité», et quiconque n'est pas un homme blanc ne pourrait plus succomber à l'intention malveillante qui se cache derrière tous les appels à une patrie américaine partagée.

$M$ ais qui aurait les meilleures chances de bien s'en sortir dans cette Amérique post-nationale? Si les minorités ethniques ou visibles et les autres groupes traditionnellement opprimés renoncent à toute identité nationale partagée avec les autres A méricains, ces derniers feront de même. La nation reposant sur des représentations imaginées communes, si une part importante de ce qui avait été jusque là une nation décidait désormais de les récuser, ce qui resterait de cette nation ne pourrait continuer comme avant. $M$ ais s'il n'y avait plus de nation américaine, sur quelle base pourrait-on s'attendre à ce que la situation s'améliore pour ceux qui étaient le moins bien traités Iorsqu'il y en avait une? Les asymétries de pouvoir ne disparaîtraient pas simplement du fait que tous soient d'accord sur la futilité d'exiger de l'État qu'il soit juste. Et personne ne pourrait rationnellement supposer que le sens de la justice des Américains économiquement et culturellement privilégiés qui était, selon Gerstle, déjà si faiblement apparent lorsqu'il y avait une nation partagée se mettrait à fleurir lorsqu'il n'y en aurait plus. Je ferai donc l'hypothèse charitable que Gerstle ne fait pas une telle supposition. Peut-être croit-il qu'il serait du moins plus difficile pour les oppresseurs traditionnels d'exploiter des gens qui étaient autrefois les membres d'une même nation dès lors qu'ils ne le sont plus. $M$ ais cela n'est guère plus éclairant. La facilité avec laquelle les États puissants ont toujours été en mesure d'abuser des minorités nationales relativement faibles et pauvres montre qu'il n'y a rien à gagner à abandonner une nationalité commune. La fin d'un sens partagé de la nationalité détruirait tout simplement le lien fragile d'appartenance, et avec lui la possibilité de tout usage de ce lien en tant qu'instrument pour un changement politique vers la justice.

Pourtant même si Gerstle semble curieusement aveugle aux conséquences d'une A mérique post-nationale, il ne s'ensuit pas que sa lecture radicalement pessimiste du passé civique de l'A mérique soit sans mérite. La vérité n'est pas toujours édifiante en histoire comme ailleurs. Peut-être que très peu d'historiens américains considèreraient la construction de la nation américaine sous un oeil aussi pessimiste que Gary Gerstle. Ce que cet exemple démontre cependant, c'est que l'histoire nationale démythifiée n'est pas un véhicule aussi fiable pour l'enthousiasme patriotique que $\mathrm{N}$ ash le suppose. Pour un grand nombre 
de gens, un passé américain démythifié ne contiendra aucun récit encourageant d'une progression morale constante selon les standards mêmes que pourrait offrir le cadre interprétatif de $\mathrm{N}$ ash de la liberté pour tous.

Pour plusieurs enfants, l'histoire multiculturelle peut développer le sentiment que la nation n'est pas une chose digne d'aucune passion civique, en dépit des meilleurs intentions des enseignants ou de ceux qui rédigent les manuels. Trop de torts ont été commis en son nom, et trop de luttes ont détruit des vies au nom d'idéaux souvent proclamés mais dont la réalisation n'a jamais été qu'imparfaite. Peut-être la meilleure chose à faire est-elle de vivre sans tenir compte de la nation. La prudence et le respect des autres nous rendra respectueux des lois, et plusieurs occasions d'aider des personnes dans le besoin se présenteront, sans qu'elles aient un lien quel conque avec l'idéal grandiose de la nation. La saisie de ces opportunités apportera à coup sûr un bénéfice tangible dans la vie des autres. La bonne volonté ne sera pas perdue dans des luttes politiques qui donnent toujours moins de résultats que ce qu'elles promettent et qui ne conduisent souvent qu'à des échecs. Faire du bénévolat dans un établissement de soins palliatifs pour sidéens ou dans un centre d'aide pour immigrants est peut-être un bien meilleur choix pour des gens bien intentionnés qui veulent que leurs bonnes intentions fassent une différence, plutôt que d'aider bénévolement un candidat à gagner une élection.

Contrairement aux critiques de droite, l'inquiétude que j'exprime ici ne concerne pas la crainte d'une nouvelle balkanisation multiculturelle. Si la meilleure histoire multiculturelle montre une chose, c'est que l'A mérique a toujours été en fait plus balkanisée que plusieurs citoyens ne voudraient l'admettre, et si on veut chercher les récents agents de la balkanisation, ceux qui ont bloqué la déségrégation des écoles américaines et isolé les quartiers défavorisés doivent porter bien plus lourdement le fardeau de la responsabilité que quel ques afrocentristes immodérés. Blâmer les spécialistes et enseignants du multiculturalisme d'avoir balkanisé la nation est un cas flagrant de prendre pour cible le messager. M a préoccupation est toute autre : I'histoire multiculturelle dans nos écoles pourrait facilement contribuer aux processus mêmes de démoralisation politique qui menacent déjà la démocratie américaine.

Les institutions d'une communauté démocratique peuvent être déstabilisées de plus d'une façon. L'inquiétude de $M$ adison qu'elles puissent succomber aux dommages causés par les factions est celle qui a traditionnellement préoccupé les A méricains. $M$ ais une autre possibilité est que ce soit moins les passions des factions que le cynisme croissant et le désengagement qui puissent défaire une communauté démocratique commune. Les gens s'imagineraient de moins en moins comme faisant partie d'une telle communauté, et se retireraient plutôt dans n'importe quel groupe qui leur donne le sentiment d'une appartenance personnelle signifiante. La réconciliation nationale deviendrait un projet que l'on abandonne, non pas dans le feu du ressentiment et de l'indignation, mais dans le retrait sans passion hors de la sphère publique. 


\section{6 - Philosophiques / Automne 2002}

6

A rrivés à ce point, nous pourrions sembler être dans une impasse. D 'une part, les citoyens américains doivent comprendre l'histoire de leur nation comme étant multiculturelle pour que la réconciliation raciale soit jamais possible. Pourtant les récits qui constituent leur histoire multiculturelle peuvent être trop complexes moralement pour les inspirer de façon fiable, et dans certains cas, elle peut être trop honteuse pour justifier autre chose que le dégoût. Les héros perdent leur statut quasi-divin et deviennent des êtres humains ambigus et souvent difficiles à cerner. Tout comme le reste d'entre nous, ils sembleront admirables par certains aspects, mais souvent horrifiants par d'autres. Aucun plan linéaire d'un progrès démocratique inexorable n'est décelable à travers le temps ou, du moins, plusieurs seront raisonnablement portés à le croire. D ans ces circonstances, un patriotisme démocratique peut sembler au mieux une réalisation précaire. D'autre part, la perte de tout sens d'une nationalité commune ne peut que causer du tort à ceux qui ont souffert le plus des idéaux discriminatoires de l'identité civique qui ont dominé sans conteste dans le passé et qui projettent leurs ombres sur le présent et le futur. Une Amérique post-nationale ne recèle aucun espoir de leur donner la justice et la reconnaissance auxquelles ils aspirent.

S'il y avait moyen de contourner cette impasse, cela nécessiterait que nous réfléchissions davantage à ce que nous devons apporter à l'interprétation du passé pour qu'une histoire patriotique puisse être possible dans les circonstances de la réconciliation. Je suggère que ce qui doit être apporté consiste spécifiquement dans des vertus morales plutôt qu'intellectuelles, même si leur pratique n'a pas à inhiber les vertus intellectuelles. Bien évidemment, nous devons nous engager en faveur des idéaux de citoyenneté libre et égale qui participent aux institutions de la démocratie libérale. En l'absence de cet engagement, personne ne peut rendre compte adéquatement d'un point de vue moral des maux que la réconciliation serait appelée à dépasser. La profondeur de cet engagement est un facteur critique s'agissant de savoir si les futurs citoyens se dévoueront aux tâches civiques de la réconciliation, tout en sachant que le succès n'est d'aucune façon garanti à l'avance. A près tout, renoncer à cette tâche simplement parce que les perspectives de succès sont toujours incertaines dans une mesure appréciable trahit un attachement décidément anémique aux valeurs que sa réussite permettrait de réaliser. $M$ ais un autre facteur entre en ligne de compte : il s'agit d'un type de détermination morale - une combinaison de courage et de patience dans la poursuite de buts auxquels nous tenons et dont la réalisation est toujours contingente, fragile et partielle. Cette détermination est quelque chose qui devrait venir facilement à ceux qui ont bénéficié le plus de la nation telle qu'elle est actuellement constituée, et s'ils peuvent affronter les circonstances de la réconciliation avec une sincérité morale, elle peut être plus facile à atteindre parmi ceux qui ont le plus souffert ${ }^{16,17}$.

16. Je remercie D ominique L eydet ainsi qu'un évaluateur anonyme pour leurs commentaires utiles sur une version antérieure.

17. Cet article a été traduit de l'anglais par M élissa Thériault. 